\title{
Erratum to: Thoracic and cardiovascular surgery in Japan during 2013
}

\author{
Annual report by The Japanese Association for Thoracic Surgery
}

\author{
Committee for Scientific Affairs, The Japanese Association for Thoracic Surgery ${ }^{1}$ • \\ Munetaka Masuda $^{2}$ Hiroyuki Kuwano ${ }^{3} \cdot$ Meinoshin Okumura $^{4} \cdot$ Hirokuni Arai $^{5}$. \\ Shunsuke Endo ${ }^{6}$ - Yuichiro Doki ${ }^{7}$ Junjiro Kobayashi ${ }^{8}$ - Noboru Motomura ${ }^{9}$. \\ Hiroshi Nishida $^{10}$ - Yoshikatsu Saiki ${ }^{11}$ Fumihiro Tanaka ${ }^{12}$ - Kazuo Tanemoto ${ }^{13}$. \\ Yasushi Toh $^{14} \cdot$ Hiroyasu Yokomise ${ }^{15}$
}

Published online: 16 June 2016

(C) The Japanese Association for Thoracic Surgery 2016

\section{Erratum to: Gen Thorac Cardiovasc Surg (2015) 63:670-701 DOI 10.1007/s11748-015-0590-3}

The following errors appeared in the above-cited article.

(A) Cardiovascular surgery section: in the 3rd paragraph, on the 4th line, "9168 cases in 2003" should read "9165 cases in 2003"; on the 6th line, " 15,757 cases in thoracic aortic aneurysm" should read " 15,758 cases in thoracic aortic aneurysm"; on the 8th line, "4.0, 4.6 and $14.6 \%$, respectively" should read " $4.0,4.7$ and $14.6 \%$, respectively"; on the 10th line, "16,752 cases" should read "16,560 cases"; and on the 13th line, "83.4\% increase"

The online version of the original article can be found under doi:10.1007/s11748-015-0590-3.

Munetaka Masuda

survey-adm@umin.net

Tokyo, Japan

2 Department of Surgery, Yokohama City University, Yokohama, Japan

3 Department of General Surgical Science, Division of Biosystem Medicine, Subdivision of Oncology, Course of Medical Sciences, Gunma University Graduate School of Medicine, Gunma, Japan

4 Department of General Thoracic Surgery, Osaka University Graduate School of Medicine, Osaka, Japan

5 Department of Cardiovascular Surgery, Tokyo Medical and Dental University Graduate School of Medical and Dental Sciences, Tokyo, Japan

6 Department of Thoracic Surgery, Jichi Medical University, Tochigi, Japan

7 Department of Gastroenterological Surgery, Osaka University Graduate School of Medicine, Osaka, Japan should read " $83.8 \%$ increase". In the 5th paragraph, on the 14th line, "6.7\% in 2003" should read "6.9\% in 2003 ". In the 6th paragraph, on the 2nd line, "83.4\%" should read " $83.8 \%$ "; on the 4th line, "placement" should read "replacement", and "2.2 and $3.7 \%$ " should read "2.9 and $5.4 \%$ "; on the 6th line, " $0.8 \%$ " should read " $1.1 \%$ "; on the 25 th line, "38.2\%" should read "38.3\%"; and on the 26th line, "41.9\%" should read " $59.4 \%$ ". In the 7th paragraph, on the 11th line, "23.4\%" should read "22.4\%". In the 8th paragraph, on the 3rd line, "1.0 and $1.7 \%$ " should read " 0.8 and $1.4 \%$ "; on the 10 th line, "5.5\%" should read "7.3\%"; and on the 14th line, "8.5-6.4\%" should read "8.0-6.4\%". In the 9th paragraph, on the 2nd line, "414 operations" should read "840 operations"; and on the 4th line, "298 operations" should read "371 operations". In the 11th paragraph, on the 6th

8 Department of Cardiovascular Surgery, National Cerebral and Cardiovascular Center, Osaka, Japan

9 Department of Cardiovascular Surgery, Toho University, Sakura Medical Center, Chiba, Japan

10 Department of Cardiovascular Surgery, The Heart Institute of Japan, Tokyo Women's Medical University, Tokyo, Japan

11 Division of Cardiovascular Surgery, Tohoku University Graduate School of Medicine, Miyagi, Japan

12 Second Department of Surgery, University of Occupational and Environmental Health, Fukuoka, Japan

13 Department of Cardiovascular Surgery, Kawasaki Medical School, Okayama, Japan

14 Department of Gastroenterological Surgery, National Kyushu Cancer Center, Fukuoka, Japan

15 Department of General Thoracic Surgery, Faculty of Medicine, Kagawa University, Kagawa, Japan 


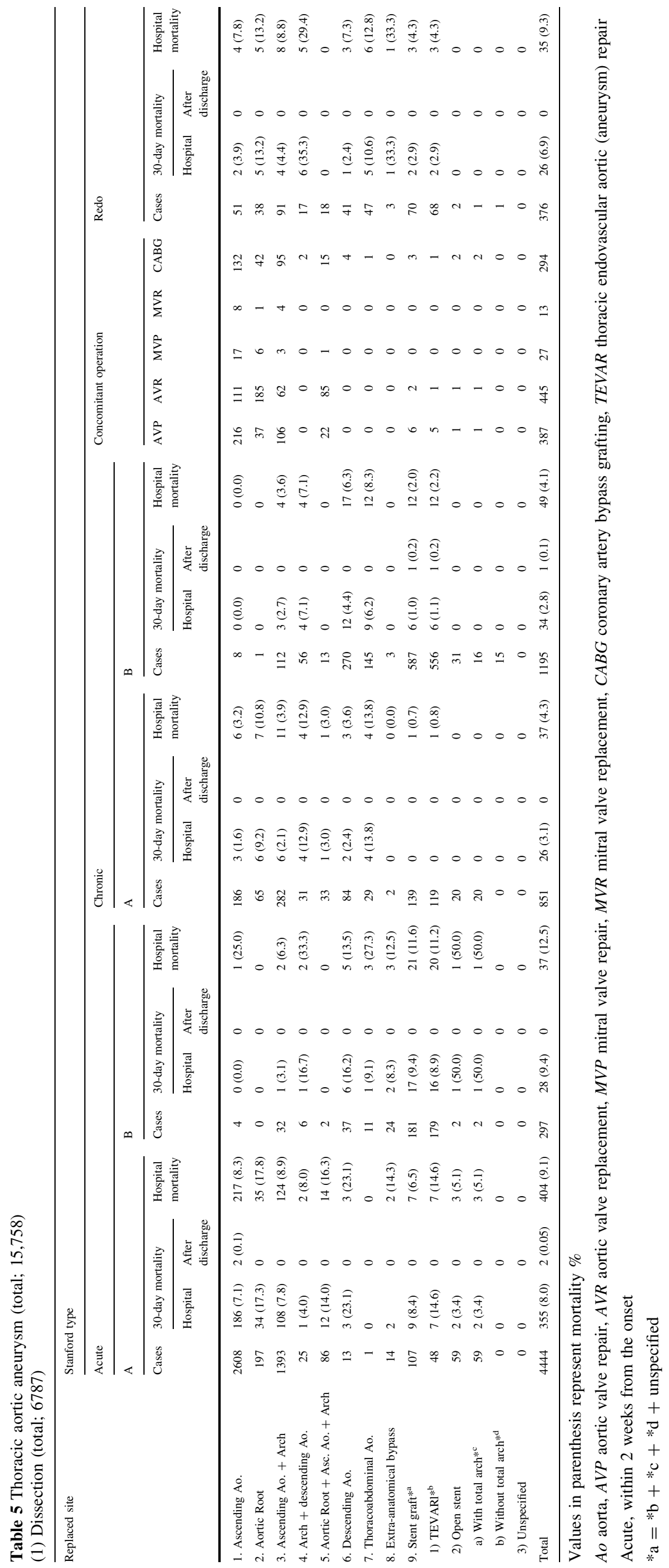


Table 7 Assisted circulation (total; 1713)

\begin{tabular}{|c|c|c|c|c|c|c|c|c|c|c|c|c|c|c|c|}
\hline \multirow[t]{4}{*}{ Sites } & \multicolumn{9}{|l|}{ VAD } & \multicolumn{6}{|c|}{ Heart-lung assist } \\
\hline & \multicolumn{3}{|l|}{ Device } & \multicolumn{6}{|l|}{ Results } & \multicolumn{2}{|c|}{ Method } & \multicolumn{4}{|l|}{ Results } \\
\hline & \multirow[t]{2}{*}{ Centrifugal } & \multirow[t]{2}{*}{ VAS } & \multirow[t]{2}{*}{ Others } & \multicolumn{3}{|c|}{ Not weaned } & \multicolumn{3}{|c|}{ Weaned } & \multirow[t]{2}{*}{ PCPS } & \multirow[t]{2}{*}{ Others } & \multicolumn{2}{|l|}{ Not weaned } & \multicolumn{2}{|l|}{ Weaned } \\
\hline & & & & On going & Death & Transplant & Alive & Deaths & Transplant & & & Deaths & Transplant & Deaths & Alive \\
\hline \multicolumn{16}{|l|}{ Post cardiotomy } \\
\hline Left & 38 & 4 & 7 & 8 & $30(61.2)$ & 0 & 3 & $8(16.3)$ & 0 & & & & & & \\
\hline Right & 0 & 0 & 0 & 0 & 0 & 0 & 0 & 0 & 0 & & & & & & \\
\hline \multicolumn{16}{|l|}{ Biventricle } \\
\hline Left & 1 & 3 & 0 & 0 & $3(75.0)$ & 0 & 1 & 0 & 0 & 499 & 69 & $274(48.2)$ & 0 & $85(15.0)$ & 209 \\
\hline Right & 4 & 0 & 0 & & & & & & & & & & & & \\
\hline \multicolumn{16}{|c|}{ Congestive heart failure } \\
\hline Left & 50 & 41 & 92 & 112 & $38(20.8)$ & 7 & 16 & $9(4.9)$ & 0 & & & & & & \\
\hline Right & 2 & 1 & 0 & 1 & 0 & 0 & 2 & 0 & 0 & & & & & & \\
\hline \multicolumn{16}{|l|}{ Biventricle } \\
\hline Left & 5 & 22 & 3 & 5 & $14(46.7)$ & 0 & 8 & $3(10.0)$ & 0 & 685 & 29 & $360(50.4)$ & 0 & $105(14.7)$ & 249 \\
\hline Right & 18 & 11 & 1 & & & & & & & & & & & & \\
\hline Respiratory failure & & & & & & & & & & 106 & 22 & $46(35.9)$ & 0 & $16(12.5)$ & 66 \\
\hline Total & 118 & 82 & 103 & 126 & $85(28.1)$ & 7 & 30 & $20(6.6)$ & 0 & 1290 & 120 & $680(48.2)$ & 2 & $206(14.6)$ & 524 \\
\hline
\end{tabular}

Values in parenthesis represent mortality \%

$V A D$ ventricular assist devise, $V A S$ ventricular assist system, $P C P S$ percutaneous cardiopulmonary support

Table 33

14. Combined resection of neighboring organ(s)

\begin{tabular}{|c|c|c|c|c|}
\hline & \multirow[t]{2}{*}{ Cases } & \multicolumn{2}{|c|}{30 day mortality } & \multirow[t]{2}{*}{ Hospital mortality } \\
\hline & & Hospital & After discharge & \\
\hline 14. Combined resection of neighboring organ(s) & 1581 & $7(0.4)$ & $3(0.2)$ & $19(1.2)$ \\
\hline \multicolumn{5}{|l|}{ (A) Primary lung cancer (organ resected) } \\
\hline Aorta & 16 & 0 & 0 & 0 \\
\hline Superior vena cava & 40 & 0 & 0 & 0 \\
\hline Brachiocephalic vein & 12 & 0 & 0 & 0 \\
\hline Pericardium & 177 & $2(1.1)$ & 0 & $3(1.7)$ \\
\hline Pulmonary artery & 227 & $1(0.4)$ & 0 & $1(0.4)$ \\
\hline Left atrium & 45 & 0 & 0 & $1(2.2)$ \\
\hline Diaphragm & 98 & $1(1.0)$ & 0 & $1(1.0)$ \\
\hline Chest wall (including ribs) & 500 & $1(0.2)$ & 0 & $9(1.8)$ \\
\hline Vertebra & 31 & 0 & 0 & $3(9.7)$ \\
\hline Esophagus & 12 & 0 & 0 & 0 \\
\hline Total & 1158 & $5(0.4)$ & 0 & $18(1.6)$ \\
\hline \multicolumn{5}{|l|}{ (B) Mediastinal tumor (organ resected) } \\
\hline Aorta & 3 & 0 & 0 & 0 \\
\hline Superior vena cava & 69 & 0 & 0 & $1(1.4)$ \\
\hline Brachiocephalic vein & 93 & $1(1.1)$ & 0 & $1(1.1)$ \\
\hline Pericardium & 267 & $1(0.4)$ & 0 & $2(0.7)$ \\
\hline Pulmonary artery & 9 & 0 & 0 & 0 \\
\hline Left atrium & 2 & 0 & 0 & 0 \\
\hline Diaphragm & 16 & 0 & 0 & 0 \\
\hline Chest wall (including ribs) & 20 & 0 & 0 & 0 \\
\hline Vertebra & 7 & 0 & 0 & 0 \\
\hline Esophagus & 1 & 0 & 0 & 0 \\
\hline Lung & 277 & $1(0.4)$ & 0 & $1(0.4)$ \\
\hline Total & 764 & $3(0.4)$ & 0 & $5(0.7)$ \\
\hline
\end{tabular}

Values in parenthesis represent mortality \% 
line, "8171 cases" should read "8971 cases"; and on the 9th line, "2.2\%" should read " $3.2 \%$ ". In the 13th paragraph, on the 4th line, "42\% increase" should read " $4.2 \%$ increase".

Additionally, there are some errors in the following tables. Table 3 (3), on the 4th line (4 Damus-Kaye-Stansel operation), values of the columns "Neonate; Hospital mortality", "Infant; 30-day mortality; Hospital", and
"Infant; Hospital mortality" should be "1 (33.3)", "4 (8.0)", and "6 (12.0)", not "1", "4", and "6", respectively. The title for Table 9 should read "Pacemaker + ICD (total; 4660)". In (B) General thoracic surgery section, Table 33, the first line of the column "30day mortality; Hospital" should read "7 (0.4)", not "7 (1.4)". Other tables with errors and their corrections are given below. 